\title{
Intravoxel incoherent motion perfusion imaging in acute stroke: initial clinical experience
}

\author{
C. Federau $\cdot$ S. Sumer $\cdot$ F. Becce $\cdot$ P. Maeder . \\ K. O'Brien • R. Meuli $\cdot$ M. Wintermark
}

Received: 26 February 2014 / Accepted: 10 April 2014 /Published online: 17 May 2014

(C) Springer-Verlag Berlin Heidelberg 2014

\begin{abstract}
Introduction Intravoxel incoherent motion (IVIM) imaging is an MRI perfusion technique that uses a diffusion-weighted sequence with multiple $b$ values and a bi-compartmental signal model to measure the so-called pseudo-diffusion of blood caused by its passage through the microvascular network. The goal of the current study was to assess the feasibility of IVIM perfusion fraction imaging in patients with acute stroke.

Methods Images were collected in 17 patients with acute stroke. Exclusion criteria were onset of symptoms to imaging $>5$ days, hemorrhagic transformation, infratentorial lesions, small lesions $<0.5 \mathrm{~cm}$ in minimal diameter and hemodynamic instability. IVIM imaging was performed at $3 \mathrm{~T}$, using a standard spin-echo Stejskal-Tanner pulsed gradients diffusion-weighted sequence, using $16 b$ values from 0 to $900 \mathrm{~s} / \mathrm{mm}^{2}$. Image quality was assessed by two radiologists, and quantitative analysis was performed in regions of interest placed in the stroke area, defined by thresholding the apparent diffusion coefficient maps, as well as in the contralateral region.
\end{abstract}

Electronic supplementary material The online version of this article (doi:10.1007/s00234-014-1370-y) contains supplementary material, which is available to authorized users.

C. Federau $(\bowtie) \cdot$ F. Becce $\cdot$ P. Maeder $\cdot$ R. Meuli Department of Diagnostic and Interventional Radiology, Centre Hospitalier Universitaire Vaudois (CHUV) and University of Lausanne, Rue du Bugnon 46, 1011 Lausanne, Switzerland e-mail: christian.federau@chuv.ch

S. Sumer $\cdot$ M. Wintermark

Neuroradiology Division, Department of Radiology, University of Virginia, Charlottesville, VA, USA

K. O'Brien

Center for Biomedical Imaging (CIBM), University of Geneva, Geneva, Switzerland
Results IVIM perfusion fraction maps showed an area of decreased perfusion fraction $f$ in the region of decreased apparent diffusion coefficient. Quantitative analysis showed a statistically significant decrease in both IVIM perfusion fraction $f\left(0.026 \pm 0.019\right.$ vs. $\left.0.056 \pm 0.025, p=2.2 \cdot 10^{-6}\right)$ and diffusion coefficient $D$ compared with the contralateral side $\left(3.9 \pm 0.79 \cdot 10^{-4}\right.$ vs. $\left.7.5 \pm 0.86 \cdot 10^{-4} \mathrm{~mm}^{2} / \mathrm{s}, p=1.3 \cdot 10^{-20}\right)$. Conclusion IVIM perfusion fraction imaging is feasible in acute stroke. IVIM perfusion fraction is significantly reduced in the visible infarct. Further studies should evaluate the potential for IVIM to predict clinical outcome and treatment response.

Keywords Diffusion · Perfusion · Stroke · IVIM

\section{Introduction}

Perfusion imaging has multiple applications in patients with cerebrovascular diseases. It allows to increase the sensitivity for the diagnosis of stroke, to rule out stroke mimics, to assess the hemodynamic significance of carotid stenosis and to identify abnormalities in patients with transient ischemic attacks when they do not have any diffusion-weighted imaging (DWI) abnormalities. The role of perfusion imaging in the assessment of the ischemic penumbra and in the selection of acute stroke patients for revascularization therapy remains unclear [1-4], as both encouraging [5-7] and discouraging results $[8,9]$ have been obtained in using perfusion-defined penumbra as a selection criterion for therapy. Some of the difficulties encountered might be due to the fact that currently used perfusion methods might not take properly into account leptomeningeal collateral circulation, which is essential for prognosis [10], as the local arterial input function might be severely inhomogeneous due to temporal delay and dispersion effects when vascular pathologies are present [11]. Further, 
two logistic limitations for the implementation of gadolinium perfusion imaging as standard of the art imaging workup for patients suspected of acute ischemic stroke are the use of contrast agent and time. Indeed, contrast agent is contraindicated in patients with renal insufficiency, which is not uncommon in the elderly stroke population, and the evaluation of renal function costs precious time. The contrast agent hurdle may be overcome by using non-contrast-agent imaging techniques such as arterial spin labeling (ASL) [12], but this increases imaging time and minimal time to treatment is seen as the most critical predictor of positive outcome in patients with acute stroke. Furthermore, ASL might suffer more than other MRI perfusion techniques from inaccuracies in arterial arrival time [13, 14], and increase in transit time might result in an underestimation of cerebral blood flow [15].

Intravoxel incoherent motion (IVIM) imaging [16], regaining interest $[17-22,28]$, is an MRI perfusion technique that might theoretically solve some of the issues listed above. First, the IVIM technique is intrinsically local and therefore independent on the arterial input function or any dispersion effects. This unique property should allow IVIM to better take into account collateral blood flow. Second, because it requires no contrast agent (no vein puncture necessary before imaging), because perfusion and diffusion information can be acquired in a single MRI sequence, and because the post-processing requirements might be fully automated, it would permit a precious gain of time in the hyperacute stage.

The basic idea behind the IVIM method is that blood flow in the (more or less) randomly laid microvascular network produces a so-called pseudo-diffusion [22], measurable with a standard diffusion sequence if the adequate set of parameters are used (low $b$ values). Because the largest part of the "diffusion signal" does arise from thermal diffusion, a bicompartmental signal model is used to extract the "diffusive" parameters of perfusion [16]. A linear relationship between IVIM perfusion parameters and standard perfusion parameters has been derived under given assumptions [22], and in particular for this study, the IVIM perfusion fraction $f$ (the percentage of total signal arising from the microvascular compartment) relates linearly with cerebral blood volume and can be understood as the ("to the pseudo-diffusion participating") microvascular cerebral blood volume.

To our knowledge, only one study of IVIM perfusion fraction measurement in acute ischemic stroke in human patients has been reported more than 15 years ago [23], without maps, and with somewhat counterintuitive quantitative results, the majority of $f$ values being reported were negative in the infarcted areas. In the context of a regain of clinical interest in IVIM as a method for measuring brain perfusion, we (re)evaluated the feasibility of IVIM perfusion fraction measurement in acute stroke.

\section{Materials and methods}

Terminology

In this report, we use interchangeably the term ischemic core (defined as the region of irreversible brain damage) and region of restricted diffusion (defined as a reduction of apparent diffusion coefficient (ADC) in comparison to adjacent brain parenchyma), although it is known that in a small number of cases, reversibility of diffusion restriction has been demonstrated [24].

\section{Patient demographics}

This bi-centric study was approved by both local ethics committees at the Universities of Virginia and Lausanne. Images were collected from February 2011 to August 2013 in patients presenting with symptoms of hemispheric acute stroke. Thirty-five patients presenting with symptoms of acute hemispheric stroke were initially considered for this study. Eighteen patients were excluded because of imaging $>5$ days after symptom onset (two patients), hemorrhagic transformation (four patients), infratentorial lesions (three patients), small lesions $<0.5 \mathrm{~cm}$ in minimal diameter (nine patients) and hemodynamic instability (no patient). The final study population consisted of 17 cases (12 males, 5 females, mean age $56.2 \pm 23.4$ years, age range $4-86$ years, time between onset of symptoms to imaging $45.5 \pm 40.3 \mathrm{~h}$, admission NIHSS $7.7 \pm 5.4,6$ patients received tPA (i.v.) before IVIM imaging; Table 1).

\section{MR imaging}

Imaging was performed on 3-T MR scanners (Trio, Verio and Skyra; Siemens, Erlangen, Germany) with a 32-channel receiver head coil. A standard stroke protocol was acquired, including T1-weighted, T2-weighted, FLAIR, DWI and timeof-flight MR angiography sequences. The IVIM imaging was based on a Stejskal-Tanner diffusion-weighted spin-echo EPI pulse sequence $[25,26]$, with multiple $b$ values $(0,10,20,40$, $80,110,140,170,200,300,400,500,600,700,800,900 \mathrm{~s} /$ $\mathrm{mm}^{2}$ ), in three orthogonal directions, and the corresponding trace was calculated. The images were oriented axially with a slice thickness of $4 \mathrm{~mm}$, a FOV of $270 \times 270 \mathrm{~mm}^{2}$ and a matrix size of $225 \times 225$, yielding an in-plane resolution of $1.2 \times$ $1.2 \mathrm{~mm}^{2}$, TR of 4,000 $\mathrm{ms}$ and TE was set to minimum (89$102 \mathrm{~ms}$, depending on the scanner). Parallel imaging was used, with an acceleration factor of 2 and a $75 \%$ partial Fourier encoding. Receiver bandwidth was 1,106 Hz/pixel, and fat was suppressed with a spectrally selective saturation routine. Acquisition time of IVIM images was 3 minutes 7 seconds. 
Table 1 Patient demographics

\begin{tabular}{|c|c|c|c|c|c|c|c|}
\hline Patient no. & Age & Sex & Stroke localization & Side & $\begin{array}{l}\text { Onset to } \\
\text { imaging }\end{array}$ & $\begin{array}{l}\text { Admission } \\
\text { NIHSS } \\
\text { score }\end{array}$ & tPA i.v. \\
\hline 1 & 42 & $\mathrm{~F}$ & $\begin{array}{l}\text { Gyrus frontalis medius, nuclei } \\
\text { caudatus and lenticularis }\end{array}$ & Right & 1 day & 8 & No \\
\hline 2 & 83 & M & $\begin{array}{l}\text { Nucleus caudatus, anterior insula } \\
\text { and frontal operculum }\end{array}$ & Left & 2 days & 6 & No \\
\hline 3 & 70 & M & Centrum semiovale & Left & $21 \mathrm{~h}$ & 14 & Yes \\
\hline 4 & 32 & M & $\begin{array}{l}\text { Gyri angularis, post-centralis and } \\
\text { temporalis superior }\end{array}$ & Left & 1 day & 13 & Yes \\
\hline 5 & 86 & M & Arteria cerebri media territory & Right & $23 \mathrm{~h}$ & 6 & No \\
\hline 6 & 79 & M & Arteria cerebri posterior territory & Left & 5 days & 12 & Yes \\
\hline 7 & 68 & M & Parietal lobe & Left & 1 day & 2 & No \\
\hline 8 & 25 & $\mathrm{~F}$ & Arteria cerebri media territory & Right & 5 days & 12 & Yes \\
\hline 9 & 63 & $\mathrm{~F}$ & Thalamus & Left & 5 days & 1 & No \\
\hline 10 & 26 & M & $\begin{array}{l}\text { Lenticulostriate and gyrus } \\
\text { temporal superior }\end{array}$ & Right & 3 days & 7 & Yes \\
\hline 11 & 58 & M & Occipital lobe and hippocampus & Left & 3 days & 0 & No \\
\hline 12 & 43 & M & Basal ganglia & Left & $10 \mathrm{~h}$ & 2 & No \\
\hline 13 & 73 & $\mathrm{~F}$ & Arteria cerebri media territory & Right & $1 \mathrm{~h}$ & 11 & No \\
\hline 14 & 4 & M & Arteria cerebri media territory & Left & 1 day & N/A & No \\
\hline 15 & 65 & $\mathrm{~F}$ & Basal ganglia and corona radiata & Right & 1 day & 3 & No \\
\hline 16 & 65 & M & Gyrus frontalis inferior & Left & 1 day & 7 & No \\
\hline 17 & 73 & M & Arteria cerebri media territory & Right & $9 \mathrm{~h}$ & 19 & Yes \\
\hline
\end{tabular}

\section{Image reconstruction}

Parametric maps of the IVIM parameters (perfusion fraction $f$, diffusion coefficient $D$, pseudo-diffusion coefficient $D^{*}$ ) were obtained by fitting the IVIM bi-exponential model [16] on a voxel-by-voxel basis, using the Levenberg-Marquardt algorithm [27], as previously described [28]. Values with $f$ greater than 0.3 and smaller than 0 were set (arbitrarily) to zero because they are not physiologic and are likely to result either from noise or from partial volume with the cerebrospinal fluid (CSF).

\section{Quantitative analysis}

In the first set of measurements, region of interests (ROIs) in the ischemic core were obtained by thresholding the ADC map [29], on a single axial slice with the largest area of infarction. The ROIs were then controlled by a neuroradiologist (CF, 4 years of experience) and manually corrected if necessary. A ROI was then placed in the contralateral region. All ROIs were placed so that they included as little CSF or large vessels as possible. The signal was then averaged for each $b$ value before fitting the IVIM bi-exponential model. To evaluate intraobserver reproducibility, these ROIs were measured twice at a 4-week interval, and the results were averaged for subsequent analysis. In the second set of measurements, the full volume of the ischemic core and the full volume with reduced $f$ were obtained, including all slices.

\section{Qualitative image evaluation}

Two experienced readers ( $\mathrm{CF}$ and FB, 9 years of experience) reviewed the IVIM perfusion fraction maps. The readers also had access to the DWI- and T2-weighted images. Before the analysis and in order to get used to the appearance of the IVIM perfusion fraction maps, the readers jointly reviewed and rated a selection of three examinations performed in the same fashion as for the study but not included in the study group. The images were then viewed independently and in random order. They rated the image quality, as well as the presence/ absence of perfusion deficits and areas of increased or decreased perfusion fraction $f$ adjacent to the ischemic core and in the stroke area. In patients with a perfusion fraction deficit area, they assessed any perfusion fractiondiffusion mismatch. Image quality was further scored using the following 5-point scale: excellent 5, good 4, fair 3, poor 2 and uninterpretable 1. A perfusion deficit was defined as an area with a visually significant decreased perfusion fraction $f$ when compared with the surrounding brain tissue and contralateral hemisphere. When there was a discrepancy between both readers, they reevaluated the images together and reached consensus agreement. Finally, the detectability of the perfusion 
Fig. 1 Box-and-whisker plots (median, 25th and 75th percentiles, minimum, maximum, outliers) of the quantitative analysis of the IVIM perfusion fraction and diffusion coefficient in the stroke area compared with the contralateral side. $[f]=$ scalar, $[D]=10^{-2} \mathrm{~mm}^{2} / \mathrm{s} . * p<0.05$

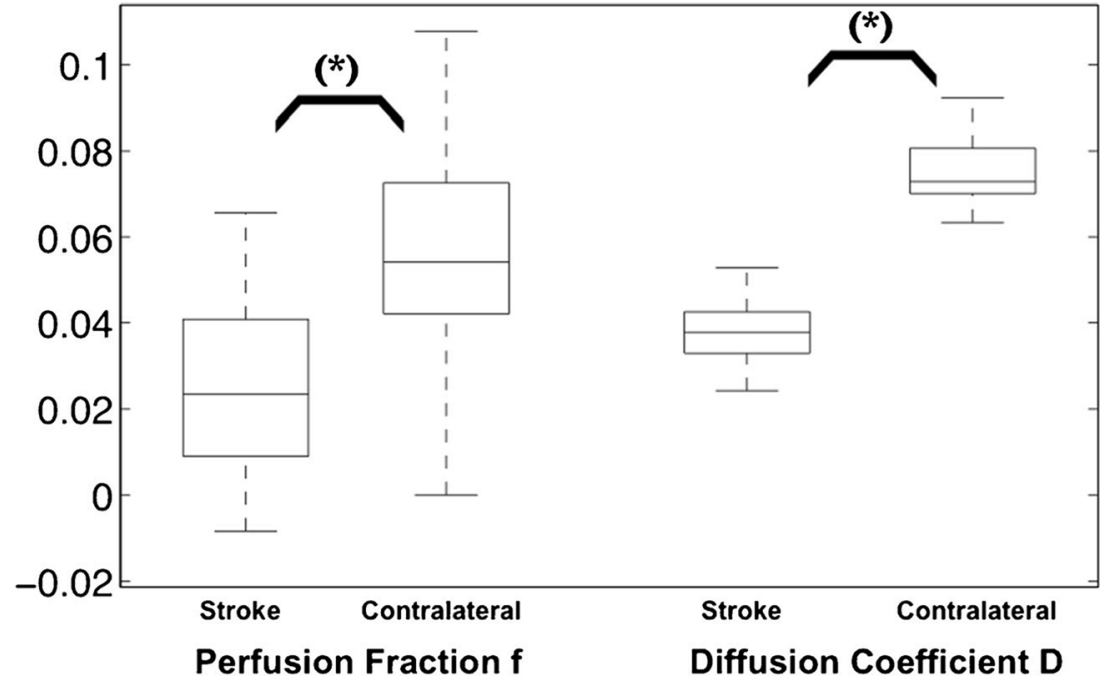

deficit was rated on the $f$ maps on a stand-alone basis by two experienced readers, first separately, followed by a consensus in case of discrepancy (CF and PM, 30 years of experience).
Statistical analysis

Data were assumed normally distributed. Two-tailed, pairwise, Student $t$ tests were calculated with Excel (Microsoft, Redmont,

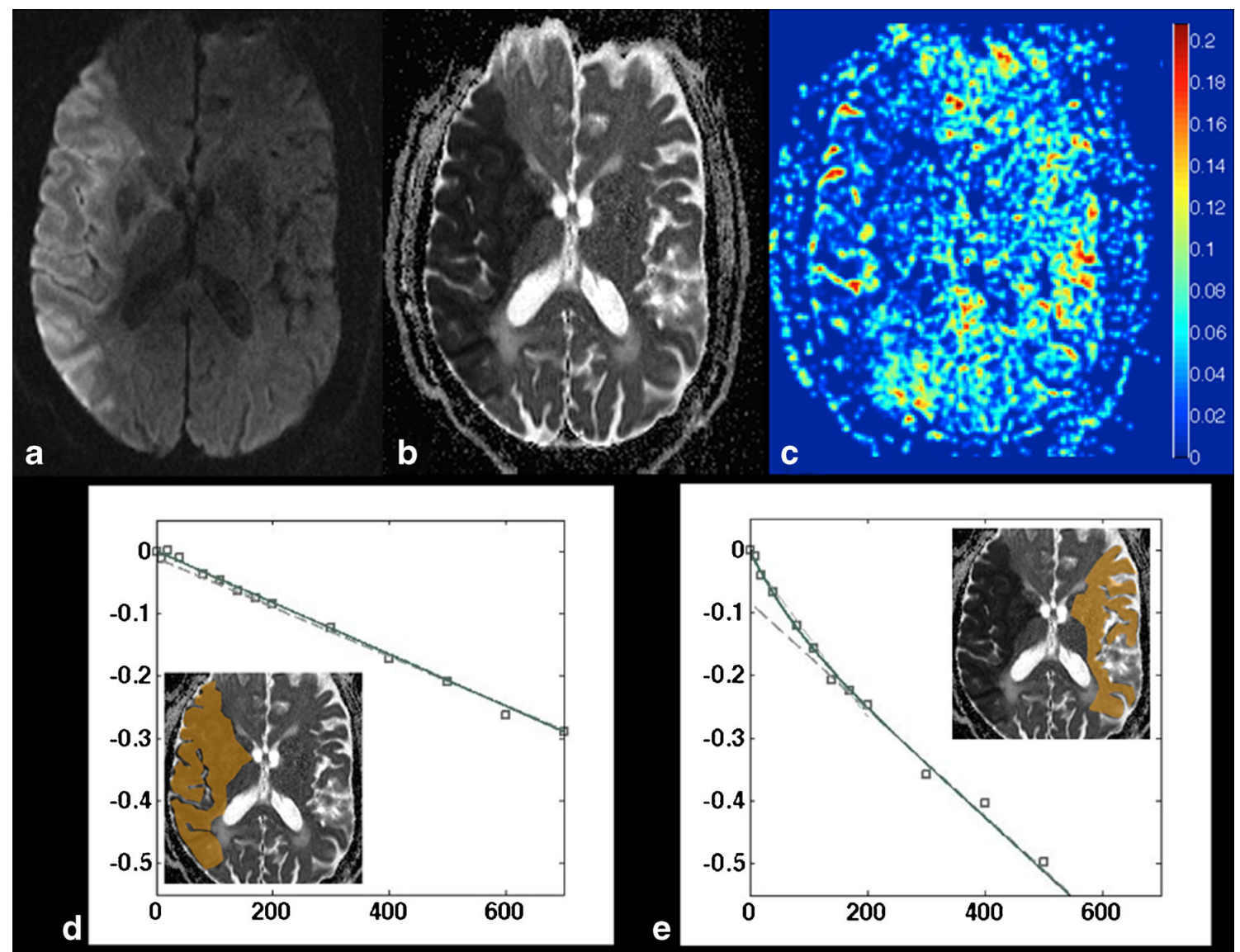

Fig. 2 Stroke in the arteria cerebri media territory on the right side, imaged $9 \mathrm{~h}$ after symptom onset, seen on the trace $\left(b=900 \mathrm{~s} / \mathrm{mm}^{2}\right)$ (a), ADC (b) and IVIM perfusion fraction $f(\mathbf{c})$. The area of reduced $f$ is slightly larger than the area of reduced ADC in the anterior region of the right frontal lobe. The "bright" regions in the stroke area of the $f$ maps are due to partial volume effects with (and/or pulsation of) the cerebrospinal fluid in the sulci. Logarithmic plot of signal intensity decay as a function of $b$, monoexponential in a ROI in the infarct core (d) and bi-exponential in a $\mathrm{ROI}$ in the contralateral region (e) 
USA), between the results obtained in the infarct core and the contralateral side, as well as between the full volume of the ischemic core and the full volume with reduced $f$. Statistical significance was defined at $p<0.05$. Intraobserver reproducibility and interobserver agreement in the quantitative analysis were evaluated with Pearson's $r$ correlation coefficient, with agreement considered to be poor $(0 \leq r \leq 0.2)$, weak $(0.2<r \leq$ $0.4)$, fair to good $(0.40<r \leq 0.75)$, or excellent $(0.75<r)$ [30]. Interobserver strength of agreement in the qualitative evaluation was estimated using the $\kappa$ coefficient and was considered without $(\kappa \leq 0)$, slight $(0<\kappa \leq 0.20)$, fair $(0.2<\kappa \leq 0.4)$, moderate $(0.4<\kappa \leq 0.6)$, substantial $(0.6<\kappa \leq 0.8)$, or almost perfect $(0.8<\kappa \leq 1)$ agreement [31].

\section{Results}

The image quality was judged fair to good $(3.59 \pm 0.38)$ with substantial agreement between both reviewers (Supplementary Table $1, r=0.64)$. Areas of decreased IVIM perfusion fraction $f$ were recognizable in $14 / 17$ patients $(\kappa=1)$ in the regions of decreased $\mathrm{ADC}$ on the produced maps. All three patients with no clearly recognizable region of decreased perfusion fraction belonged to the four smallest lesions. On the stand-alone $\mathrm{f}$ maps, the detection of the hypoperfused area was dependent on the lesion size. All large $(>7 \mathrm{ml})$ hypoperfused areas could be detected (9/9), and half of the smaller lesions were detected as well (4/8, 3 of the 4 lesions not detected had a volume smaller than $1 \mathrm{ml}$, the last one having a volume of $6.61 \mathrm{ml} ; \kappa=0.61$ ).

Quantitative analysis showed a statistically significant decrease in both IVIM perfusion fraction $f(0.026 \pm 0.019$ vs. $\left.0.056 \pm 0.025, p=2.2 \cdot 10^{-6}\right)$ and diffusion coefficient $D$ compared with the contralateral side $\left(3.9 \pm 0.79 \cdot 10^{-4}\right.$ vs. $7.5 \pm 0.86$. $10^{-4} \mathrm{~mm}^{2} / \mathrm{s}, p=1.3 \cdot 10^{-20}$; Fig. 1$)$. Intraobserver reproducibility was fair to good for $f(r=0.69)$ and excellent for $D(r=0.96)$.

The average volume of the ischemic core $(40.25 \pm$ $72.53 \mathrm{ml}$ ) was not different than the average volume of reduced perfusion fraction $(42.05 \pm 85.46 \mathrm{ml}, p=0.64)$. One patient showed a mismatch (Fig. 2, the volume of the reduced perfusion fraction was $24.76 \%$ bigger than the ischemic core; imaging was done $9 \mathrm{~h}$ after symptom onset). Three additional patients showed an incompletely overlapping ischemic core and volume of reduced $f$, without quantitative mismatch. Five patients showed an area of increased $f$ around the ischemic core (Fig. 3, $\kappa=0.82$ ). Six patients showed some focal, small areas of increased $f$ inside the ischemic core (some of those areas showed a focal increased in $D$, some did not; $\kappa=1$ ).

\section{Discussion}

This study demonstrates that diffusion and local microvascular perfusion information can be acquired in the context of

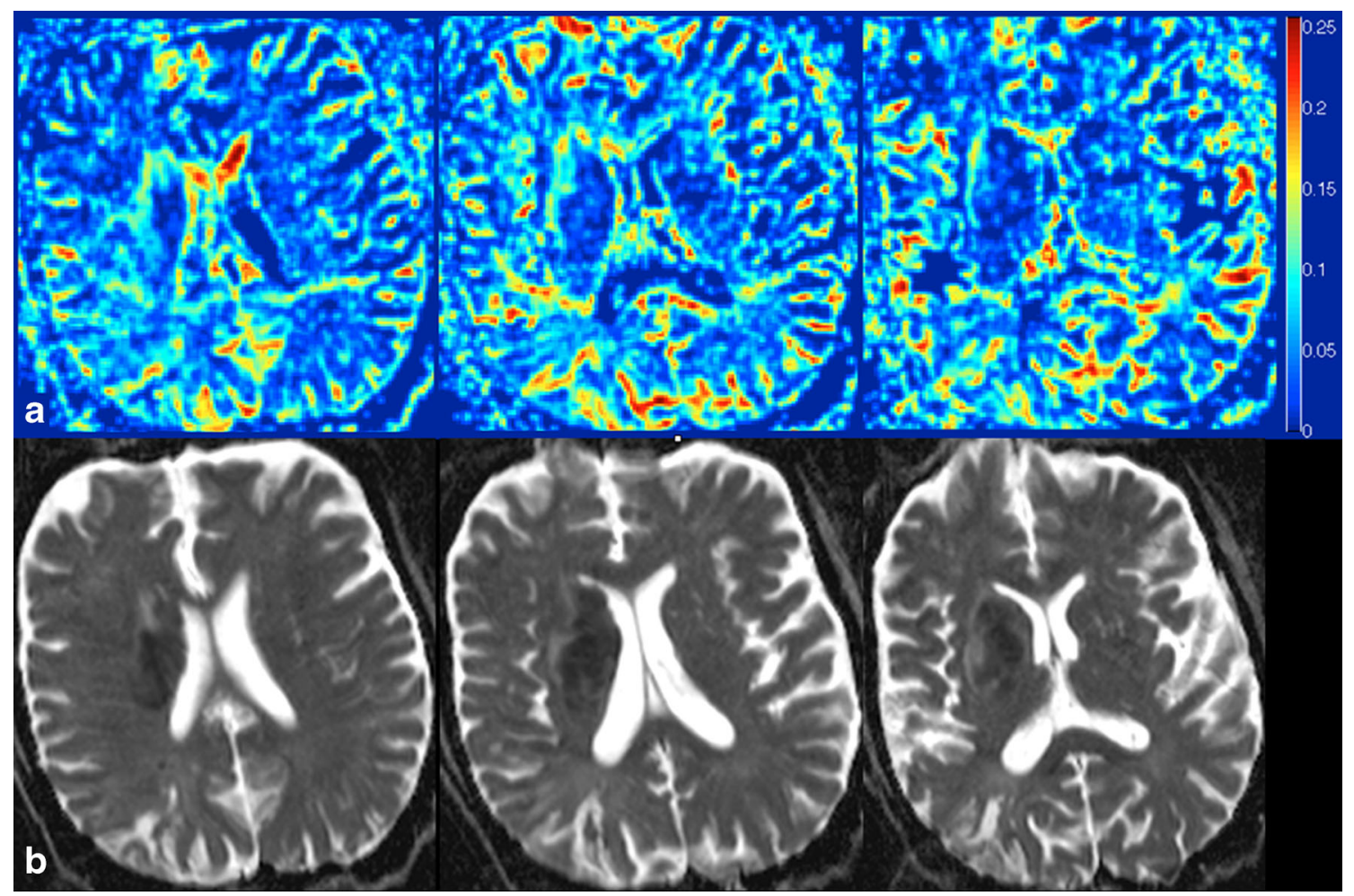

Fig. 3 Three consecutive axial slices of a stroke in the right sub-insular region, seen on IVIM perfusion fraction $f$ (a) and ADC (b). Rostrally and laterally, the ischemic core is bordered by a region of increased perfusion fraction 
acute stroke with a single, 3-min IVIM MRI sequence, without contrast agent injection. The IVIM perfusion fraction $f$ was significantly reduced in the infarct core, and the perfusion fraction maps were generally of sufficient quality to evaluate the extension of local microvascular blood volume abnormalities in the context of acute stroke. The average ischemic core and the volume of reduced perfusion fraction $f$ were not significantly different, and a (relatively small) mismatch was seen in only one patient. This might be due to the relatively late time point of imaging after stroke onset in the present study, and it would be of interest to repeat this study in a population of patients presenting earlier after symptom onset. Detection of large areas of hypoperfusion on the $f$ maps was feasible on a stand-alone basis, while signal-to-noise ratio improvements will be necessary to detect all smaller lesions.

The presented results add to recent validation reports of the method in the brain, which showed a gradual increase of the IVIM perfusion parameters to a hypercapnia challenge [28], and a dependence of those parameters on the cardiac cycle [17], as well as usefulness for the differentiation between high- and low-grade brain gliomas [19, 21], and in the assessment of treatment response of gliomas to anti-angiogenic therapy [20].

The following technical points require some special attention. We presented only the results of the perfusion fraction $f$, and not those of the pseudo-diffusion coefficient $D^{*}$, because the interpretation of the latter, representing the diffusion coefficient of a vanishing compartment, is challenging. Further, the exact subset of protons contributing to the pseudodiffusion remains to be characterized with precision, as for example partial volume effects with CSF molecules from subarachnoid as well as from the Virchow-Robin perivascular spaces might contribute to the observed double exponential decay of the signal [32]. This might explain why the perfusion fraction in the regions of acute infarction was not fully vanishing. Therefore, an IVIM acquisition suppressing CSF signal might be of interest. A standard $180^{\circ}$-inversion recovery pulse has the major drawback of also strongly suppressing blood signal. Alternative pulse sequences with better blood recovery under CSF suppression are currently under investigation, such as using a T2 magnetization preparation [33]. Furthermore, the accuracy of the fitting method is dependent among other things on signal-to-noise (itself dependent on the $b$ value as well as on the position of the voxel in the brain), as well as on the size of the parameters themselves [34, 35]. For this reason, to optimize signal-to-noise for quantitative analysis and therefore physiological meaningfulness, the signal was first averaged in the ROIs before fitting. Further, the perfusion fraction is the T1- and T2-weighted volume fraction of incoherently flowing blood in the tissue, and therefore, including relaxation time of the various involved components might increase the accuracy of the estimation. Finally, the IVIM method assumes a homogenous local magnetic field, and therefore, "hyperperfusion" artifacts should be expected in proximity of regions containing a paramagnetic substance such as blood products or metal.

This study suffers from several limitations. The cohort studied is small, and we restricted our analysis to stroke lesions larger than $0.5 \mathrm{~cm}$ in diameter. The intrasubject reproducibility was not studied. The timing of the studies was not homogenous. No comparison with other perfusion method was performed. Not all patients were treated with i.v. tPA, and the recanalization status was not assessed.

The IVIM method to measure brain perfusion deserves more attention. IVIM measures signal arising mainly from the microvasculature, i.e. from the vessels involved in the nutritive exchange, while DSC perfusion imaging is largely sensitive to blood flowing in larger vessels as well, which do not participate in gas exchange. Because microvascular and macrovascular blood flow might dissociate in ischemic brain tissue in ways that directly affect tissue survival $[36,37]$, this property of IVIM might have important therapeutic implication and should be further investigated.

\section{Conclusion}

This report demonstrates the feasibility of IVIM perfusion fraction imaging in the context of acute stroke.

Acknowledgement This work was supported by the Centre d'Imagerie BioMédicale (CIBM) of the UNIL, UNIGE, HUG, CHUV and EPFL and the Leenaards and Louis-Jeantet Foundations. CF was supported by the Faculté de Biologie et de Médecine of the University of Lausanne.

Ethical standards and patient consent We declare that all human and animal studies have been approved by the local ethics committees at the Universities of Virginia and Lausanne and have therefore been performed in accordance with the ethical standards laid down in the 1964 Declaration of Helsinki and its later amendments. We declare that all patients gave informed consent prior to inclusion in this study.

Conflict of interest We declare that we have no conflict of interest.

\section{References}

1. Lev MH (2013) Perfusion imaging of acute stroke: its role in current and future clinical practice. Radiology 266:22-27

2. Goyal M, Menon BK, Derdeyn CP (2013) Perfusion imaging in acute ischemic stroke: let us improve the science before changing clinical practice. Radiology 266:16-21

3. Wintermark M, Sanelli PC, Albers GW et al (2013) Imaging recommendations for acute stroke and transient ischemic attack patients: a joint statement by the American Society of Neuroradiology, the American College of Radiology, and the Society of NeuroInterventional Surgery. AJNR Am J Neuroradiol 10:828-832

4. Wintermark M, Warach SJ, STIR et al (2013) Acute stroke imaging research roadmap II and international survey of acute stroke imaging capabilities: we need your help! AJNR Am J Neuroradiol 34:1671 
5. Albers GW, Thijs VN, Wechsler L et al (2006) Magnetic resonance imaging profiles predict clinical response to early reperfusion: the diffusion and perfusion imaging evaluation for understanding stroke evolution (DEFUSE) study. Ann Neurol 60:508-517

6. Lansberg MG, Straka M, Kemp S et al (2012) MRI profile and response to endovascular reperfusion after stroke (DEFUSE 2): a prospective cohort study. Lancet Neurol 11:860-867

7. Davis SM, Donnan GA, Parsons MW et al (2008) Effects of alteplase beyond $3 \mathrm{~h}$ after stroke in the Echoplanar Imaging Thrombolytic Evaluation Trial (EPITHET): a placebo-controlled randomised trial. Lancet Neurol 7:299-309

8. Hacke W, Furlan AJ, Al-Rawi Y et al (2009) Intravenous desmoteplase in patients with acute ischaemic stroke selected by MRI perfusion-diffusion weighted imaging or perfusion CT (DIAS-2): a prospective, randomised, double-blind, placebocontrolled study. Lancet Neurol 8:141-150

9. Kidwell CS, Jahan R, Gornbein J et al (2013) A trial of imaging selection and endovascular treatment for ischemic stroke. N Engl J Med 368:914-923

10. Menon BK, Smith EE, Modi J et al (2011) Regional leptomeningeal score on CT angiography predicts clinical and imaging outcomes in patients with acute anterior circulation occlusions. AJNR Am J Neuroradiol 32:1640-1645

11. Calamante F, Willats L, Gadian DG et al (2006) Bolus delay and dispersion in perfusion MRI: implications for tissue predictor models in stroke. Magn Reson Med 55:1180-1185

12. Chalela JA, Alsop DC, Gonzalez-Atavales JB et al (2000) Magnetic resonance perfusion imaging in acute ischemic stroke using continuous arterial spin labeling. Stroke 31:680-687

13. Calamante F, Williams SR, van Bruggen N et al (1996) A model for quantification of perfusion in pulsed labelling techniques. NMR Biomed 9:79-83

14. Alsop DC, Detre JA (1996) Reduced transit-time sensitivity in noninvasive magnetic resonance imaging of human cerebral blood flow. J Cereb Blood Flow Metab 16:1236-1249

15. Bokkers RP, van Laar PJ, van de Ven KC et al (2008) Arterial spinlabeling MR imaging measurements of timing parameters in patients with a carotid artery occlusion. AJNR Am J Neuroradiol 29:16981703

16. Le Bihan D, Breton E, Lallemand D et al (1988) Separation of diffusion and perfusion in intravoxel incoherent motion MR imaging. Radiology 168:497-505

17. Federau C, Hagmann P, Maeder P, et al. (2013) Dependence of brain intravoxel Incoherent motion perfusion parameters on the cardiac cycle. PLoS One 8:e72856

18. Federau C, O'Brien K, Meuli R et al (2014) Measuring brain perfusion with intravoxel incoherent motion (IVIM): initial clinical experience. J Magn Reson Imaging 39:624-632
19. Federau C, Meuli R, O’Brien K et al (2014) Perfusion measurement in brain gliomas with intravoxel incoherent motion MRI. AJNR Am J Neuroradiol 35:256-262

20. Kim HS, Suh CH, Kim N et al (2014) Histogram analysis of intravoxel incoherent motion for differentiating recurrent tumor from treatment effect in patients with glioblastoma: initial clinical experience. AJNR Am J Neuroradiol 35:490-497

21. Bisdas S, Koh TS, Roder C et al (2013) Intravoxel incoherent motion diffusion-weighted MR imaging of gliomas: feasibility of the method and initial results. Neuroradiology 55:1189-1196

22. Le Bihan D, Turner R (1992) The capillary network: a link between IVIM and classical perfusion. Magn Reson Med 27:171-178

23. Wirestam R, Brockstedt S, Lindgren A et al (1997) The perfusion fraction in volunteers and in patients with ischaemic stroke. Acta Radiol 38:961-964

24. Fiehler J, Foth M, Kucinski T et al (2002) Severe ADC decreases do not predict irreversible tissue damage in humans. Stroke 33:79-86

25. Stejskal EO, Tanner JE (1965) Spin diffusion measurements: spin echoes in the presence of a time-dependent field gradient. J Chem Phys 42:288-292

26. Turner R, Le Bihan D, Maier J et al (1990) Echo-planar imaging of intravoxel incoherent motion. Radiology 177:407-414

27. Seber GA, Wild CJ (2003) Nonlinear regression. Wiley, Hoboken

28. Federau C, Maeder P, O'Brien K et al (2012) Quantitative measurement of brain perfusion with intravoxel incoherent motion MR imaging. Radiology 265:874-881

29. Schaefer PW, Copen WA, Lev MH et al (2005) Diffusion-weighted imaging in acute stroke. Neuroimaging Clin N Am 15:503-530, ix-x

30. Fleiss JL (1986) The design and analysis of clinical experiments. Wiley, New York

31. Landis JR, Koch GG (1977) The measurement of observer agreement for categorical data. Biometrics 33:159-174

32. Kwong KK, McKinstry RC, Chien D et al (1991) CSF-suppressed quantitative single-shot diffusion imaging. Magn Reson Med 21:157-163

33. Federau C, O'Brien K (2014) Increased IVIM perfusion contrast in the brain with T2 magnetization preparation. \# 3369, Proc Int Soc Magn Res Med, Milan

34. Pekar J, Moonen T, Van Zijl P (1992) On the precision of diffusion/ perfusion imaging by gradient sensitization. Magn Reson Med 23: $122-129$

35. King MD, vam Bruggen N, Busza AL (1992) Perfusion and diffusion MR imaging. Magn Reson Med 24:288-301

36. Tso MK, McDonald RL (2013) Acute microvascular changes after subarachnoid hemorrhage and transient global cerebral ischemia. Stroke Res Treat 2013:425281

37. Theilen H, Schröck H, Kuschinsky W (1993) Capillary perfusion during incomplete forebrain ischemia and reperfusion in rat brain. Am J Physiol 265:H642-H648 